\title{
Dynamics of pulsed flow in an elastic tube
}

\author{
${ }^{1}$ Amit K. Chattopadhyay and ${ }^{2}$ Shreekantha Sil \\ ${ }^{1}$ Max Planck Institute for the Physics of Complex Systems, Nöthnitzer Straße 38, 01187 Dresden, Germany \\ ${ }^{2}$ Department of Physics, Indian Institute of Science, Bangalore 560012, India *
}

\begin{abstract}
Internal haemorrhage, often leading to cardio-vascular arrest happens to be one of the prime sources of high fatality rates in mammals. We propose a simplistic model of fluid flow to specify the location of the haemorrhagic spots, which, if located accurately, could be operated upon leading to a possible cure. The model we employ for the purpose is inspired by fluid mechanics and consists of a viscous fluid, pumped by a periodic force and flowing through an elastic tube. The analogy is with that of blood, pumped from the heart and flowing through an artery or vein. Our results, aided by graphical illustrations, match reasonably well with experimental observations.
\end{abstract}

PACS numbers: 87.10.+e, 87.27.As, 87.45.-k, 47.10.+g

\section{INTRODUCTION}

The dynamics of fluid flow through elastic tubes occur in many natural processes, like the organic respiration process, fluid flow in spongy bodies and flow of blood in cardiac systems. Studies of these phenomena have covered a substantial portion of the literature of biological systems [1-15]. A typical example is the flow of blood through arteries and veins which are not rigid tubes but elastic tubes.

These real life systems have been the objects of investigation for quite a while now and in many of these processes, nonlinear instabilities have been observed to occupy positions of paramount importance [8, [9], often something which can be linked with the likes of Rayleigh or the "pearling" instability seen in tubular lipid membranes [10]. Presently, the major emphasis on cardiac related studies seem to be on the electrophysiology of cardiac tissues during ischemic jolts 13 and attempts on linking different aperiodic cardiac processes to studies in chaos and turbulence 15, 17, 22, 23. . Other studies have focussed on the time series analysis of the vibrating heart 11, 12, 19, 21] with efforts on explaining the pulsating dynamics of cardiac muscles in the more physically quantifiable language of chaos and associated instabilities. Works in a somewhat different line have shown that applying fundamental principles of nonlinear physics, it is possible to forecast beforehand the cardiac disarray arising due to rapid heart rates (ventricular tachycardia (VT)) 11 or that due to aperiodic beating of the heart (ventricular fibrillation (VF)) 11, 12. Of the wide range of possibilities for their origin, researches have been focussed on the transition from tachycardia to fibrillation, generating spiral waves 13, 15, 16] to the anisotropic nature of ventricles 14 leading to faster electrical propagation parallel to the long axis of muscle fibers. The

\footnotetext{
*Present address: Department of Physics, Viswa Bharati Univer-
} sity, Santiniketan, West Bengal, India different methods of electrical defibrillation which have been presently proposed 15, 18, 19 bear testimony to the intense efforts on arriving at medical solutions from theoretical studies of bio-systems. In this paper, we attempt to have an understanding of a complex biological process, that of internal haemorrhage in an artery or vein, through comparisons with an analogical mechanical model. It is to be noted that our approach is much more generalized in nature than that due to Sinha, et al [15] or by Panfilov, et al [18], in the sense that we not only consider the case of ventricular fibrillation as a cause for aperiodicity in blood flow but we include all possible external disturbances (like a sudden thud on any portion of the body) that might lead to internal haemorrhage. As such, our approach includes the dynamics of waves propagating around a steady obstacle [19, 20] within the heart (clotted blood, in our case) as well as effectual VF. However, it needs to be mentioned that since all our conclusions would be drawn via a mapping to a much simpler mechanical system, we have no claims to exactness in terms of biological details. But as we would indicate in the end, our qualitative understanding can possibly be generalized to enable further physiological details, through a simple extension of our model. In the following analysis, the main focus of our study would be to determine the point of internal haemorrhage in a single channel flow, using fundamental principles of fluid mechanics.

This necessitates a clearer picture of the real life system in operation. Experiments performed long ago by Brecher, et al [4], on the steady flux of blood through the superior vena cava of a dog with respect to the pressure difference in the jugular vein, although initially shows a linear behavior, the flux somewhat paradoxically attains a maximum value and no longer increases. It is known for a long time that the flow of blood is pulsatile as a consequence of the beating of the heart. The beating heart produces a pressure pulse that travels through the blood, and this pressure wave is the pulse felt in arteries. However there is a marked difference between the acoustic waves generated in the heart and those which 
are felt in arteries. For the former, the waves are a consequence of the compressibility of the blood and of the living tissues surrounding it; while for the latter, the wave owes its existence to the elasticity of the arteries and the coupling of the vibrations of the artery walls to the blood flow. In 1961, Bergel [5] studied the velocity of propagation of the pulse wave through the thoracic aorta. In his derivation of the velocity of blood through arteries, he considered blood as an inviscid and incompressible fluid. Inspite of this non-realistic assumption of an inviscid fluid, which technically means that the fluid is supposed to be in the high Reynolds' number zone, he obtained a wonderful match with the experimental value of the velocity obtained by McDonald [6]. However the calculation predicted that the pulse travels undeformed during propagation, and this is in sharp contrast to experimental observations. Real life data show that the pulse wave changes its shape slowly as it travels through the major arteries. This immediately suggests that to model a real life system, it is extremely important to take into account the viscous nature of the blood. In this work, we study the deformation of a pressure wave (the heart pulse) when it propagates through a viscous fluid (the blood) flowing through an elastic tube. Our analysis reveals a wonderful set of results inherent to the viscous nature of blood and which match with the experimental observations.

\section{THE THEORETICAL MODEL}

The starting point of our analysis is the well-known Navier-Stokes' equation in fluid mechanics [25] and the equation of continuity, for the case of a viscous, compressible flow through an elastic tube. We consider an elastic tube filled with a fluid at rest and surrounded by a fluid. The radius of the tube will be determined by the transmural pressure difference between the interior and exterior pressures, as well as the tension in the wall of the tube. Under normal circumstances, the thickness of a blood vessel wall is small compared to the resting radius of the blood vessel. Consequently, to a good approximation, we can treat the wall as a thin membrane. Since the pulsating waveform causes the cross-sectional area to depend on the spatial location, let $\mathrm{A}(\mathrm{x})$ be the cross-sectional area of the tube at a distance $\mathrm{x}$, the pressure at that point, at time $\mathrm{t}$ be $\mathrm{p}(\mathrm{x}, \mathrm{t})$ and the density is taken to be $\rho$. If $\mathrm{u}(\mathrm{x}, \mathrm{t})$ be the velocity parallel to the tube axis, the continuity equation gives,

$$
\frac{\partial}{\partial t}(\rho A)+\frac{\partial}{\partial x}(\rho A u)=0
$$

The modified Navier-Stokes' equation in the absence of a perpendicular component of the velocity reduces to,

$$
\begin{aligned}
A\left[\frac{\partial}{\partial t}(\rho u)\right. & \left.+u \frac{\partial}{\partial x}(\rho u)\right]=-\frac{\partial}{\partial x}\left[\left(p-p_{0}\right) A\right] \\
& +\nu A \rho \frac{1}{r} \frac{\partial}{\partial r}\left[r \frac{\partial u}{\partial r}\right]+\nu A \rho \frac{\partial^{2} u}{\partial x^{2}}
\end{aligned}
$$

where $\mathrm{r}$ gives the radius of the tube at time $\mathrm{t}$ and $\nu$ is the Newtonian (and not the kinematic) viscosity of the fluid.

Assuming linearized pressure dependence of density, $i$. e. $p-p_{0}=c_{0}^{2}\left(\rho-\rho_{0}\right)\left(c_{0}\right.$ being the mean velocity of blood) and considering the deformable material of the walls of the blood vessels obey Hooke's law, the pressure difference across the walls of a thin membrane is given by [3],

$$
p(x)-p_{0}=\frac{E h}{r_{0}}\left(1-\frac{r_{0}}{r}\right)
$$

where E is the Young's modulus of the walls of the artery (or vein), $r_{0}$ is the mean radius and $\mathrm{h}$ is the thickness of the artery (or vein) wall. Now we address the important question, that of the relative importance of the nonlinear terms with respect to the linear ones in the above constitutive equations. A simple look at the magnitude of the Reynold's number (obtained from putting in experimental data [5]) tells us that it has a value less than 60 [26] and naturally we expect the dynamics to lie within the inertial zone. Physically speaking, incorporation of nonlinear terms would mean considering the effects of microscopic fluctuations occurring at the boundaries of the artery (or vein) walls due to the periodic input pulse. But the walls of the artery (or vein) being highly elastic (that is quite susceptible to stress), the average width of these fluctuations would be much smaller in magnitude than the mean radius $r_{0}$. Thus we can safely drop all nonlinearities henceforth. As an additional comment, it might be suggestive that this dropping of higher-ordered terms is only to aid an exact analytical solution, although it is indeed a trivial exercise to exactly solve the two coupled equations numerically. However, as has been already indicated, this would not add to the physics of mean-flow in any way.

Linearizing equations (1) and (2) and neglecting all second-ordered terms (alternative linearization schemes in the contexts of reaction-diffusion systems, which can also be mapped to fluid flows, are to be found in [27]), these respectively reduce to

$$
\rho_{0} \frac{\partial u}{\partial x}=-\beta \frac{\partial p}{\partial t}
$$

where we use the fact that $\mathrm{A}=\pi r^{2}$, and $\beta$ has the value $\beta=\frac{1}{A_{0}}\left(\frac{\rho_{0}}{\alpha}+\frac{A_{0}}{c_{0}{ }^{2}}\right), \alpha$ being equal to $\frac{E h}{2 r_{0} A_{0}}$. Eqn.(2) reduces to 


$$
\begin{aligned}
A_{0} \rho_{0} \frac{\partial u}{\partial t} & =-A_{0} \frac{\partial p}{\partial x}+\nu A_{0} \rho_{0} \frac{\partial^{2} u}{\partial r^{2}}+\frac{\nu \rho_{0} A_{0}}{r} \frac{\partial u}{\partial r} \\
& +\nu \rho_{0} A_{0} \frac{\partial^{2} u}{\partial x^{2}}
\end{aligned}
$$

It is to be noted that $\beta$ is basically independent of $c_{0}$ once the latter becomes large (which it indeed is), relative to the other term.

Now, considering the variation of the last equation with respect to $x$ and utilising equation (4), we get

$$
\begin{aligned}
\frac{\partial}{\partial t}\left(-\beta \frac{\partial p}{\partial t}\right) & =-\frac{\partial^{2} p}{\partial x^{2}}+\nu \frac{\partial^{2}}{\partial r^{2}}\left(-\beta \frac{\partial p}{\partial t}\right)+\frac{\nu}{r} \frac{\partial}{\partial r}\left(-\beta \frac{\partial p}{\partial t}\right) \\
& -\frac{\nu \rho_{0}}{r^{2}} \frac{\partial r}{\partial x} \frac{\partial u}{\partial r}+\nu \frac{\partial^{2}}{\partial x^{2}}\left(-\beta \frac{\partial p}{\partial t}\right)
\end{aligned}
$$

Rearranging and once again plugging in results from equation (4), we finally obtain the complex wave equation as

$$
\beta \frac{\partial^{2} p}{\partial t^{2}}=\frac{\partial^{2} p}{\partial x^{2}}+\gamma \frac{\partial p}{\partial t}+\lambda \frac{\partial^{2}}{\partial x^{2}}\left(\frac{\partial p}{\partial t}\right)
$$

with $\lambda=\nu \beta$ and $\gamma=\frac{3 \pi \lambda}{A_{0}}$. All quantities with suffix ' 0 ' denote the corresponding parameters for the unstretched elastic tube. In the above derivation, spatial variations in density were ignored (not exactly a Boussinesq approximation) to avoid nonlinearities. More specifically, in arriving at equation (7) from (6), we have used the relations $\frac{\partial^{2}}{\partial r^{2}}\left(\frac{\partial p}{\partial t}\right)=\frac{6 \pi}{A} \frac{\partial p}{\partial t} \approx \frac{6 \pi}{A_{0}} \frac{\partial p}{\partial t}, \frac{1}{r} \frac{\partial}{\partial r}\left(\beta \frac{\partial p}{\partial t}\right)=-\frac{2}{r^{2}} \frac{\partial p}{\partial t} \approx-\frac{2 \pi}{A_{0}} \frac{\partial p}{\partial t}$ and $\frac{\rho_{0}}{r^{2}} \frac{\partial r}{\partial x} \frac{\partial u}{\partial r}=-\frac{\beta}{r^{2}} \frac{\partial p}{\partial t} \approx-\frac{\pi \beta}{A_{0}} \frac{\partial p}{\partial t}$.

The second and third terms appearing on the right hand side of the above equation represent the damping rate due to viscosity. In fact, this dissipation is responsible for the deformation of the wave pulse and is of paramount importance in our theory. Now following usual methods, the modulated pressure wave is represented as

$$
p_{k}=p_{0} e^{-\frac{\left(\lambda k^{2}-\gamma\right)}{2 \beta} t} \times e^{i\left(\frac{1}{2 \beta} \sqrt{-\left(\gamma-\lambda k^{2}\right)^{2}+4 \beta k^{2}} t+k x\right)}
$$

Damping of the wave occurs for those Fourier components for which $k^{2} \leq \frac{\gamma}{\lambda}$. This implies that the Fourier modes with $k_{0}=\sqrt{\frac{\gamma}{\lambda}}=\frac{\sqrt{3}}{r_{0}}$ will propagate through the system without any distortion with velocity $u_{k_{0}}=\frac{1}{\sqrt{\beta}}$. Interestingly the velocity of this particular mode matches with the results obtained by Bergel [5]. The general dependence of velocity on $\mathrm{k}$ is given by

$$
u_{k}=\frac{1}{\sqrt{\beta}} \sqrt{1-\frac{\left(k^{2} \lambda-\gamma\right)^{2} \beta}{4 k^{2}}}
$$

and this implies that the propagation mode exists only within the selected range

$$
\sqrt{\frac{\gamma}{\lambda}}<|\vec{k}|<\frac{\sqrt{\beta}}{\lambda}+\frac{\sqrt{\beta+\gamma \lambda}}{\lambda}
$$

The key point in the above velocity expression is that it is, in general, highly dispersive. However, the fact that we have arrived at equation (8) utilising equations (4) and (7) precludes the possibility that in the process of Fourier transforming the measured velocity to the wave vector space, localized singularities would be lost and this means that our analysis is practically valid only for highly dispersive haemorrhages. This, though, is not a major deterrent, since almost all real haemorrhagic conditions conform to such a regime.

\section{COMPARISON WITH EXPERIMENTAL DATA AND RESULTS}

In the following we give a model estimation of the velocity of blood as calculated from our theory utilizing parameters for blood flowing through the thoracic aorta of dogs [5]. Here $E=4.3 \times 10^{6}$ dynes $/ \mathrm{cm}^{2}$, $h / r_{0}=0.105, \rho_{0}=1.06 \mathrm{gm} /$ c.c., $p_{0}=100 \mathrm{~mm}$ of $\mathrm{Hg}$ pressure, $\nu=0.035$ dyne-secs $/ \mathrm{cm}^{2}, r_{0}=0.216 \mathrm{~cm}$, $c_{0}=1571 \mathrm{~m} / \mathrm{s}$. With these values, the undamped wave velocity $\left(u_{k_{0}}=\sqrt{\frac{\gamma}{\lambda}}\right)$, calculated from our theory (4.61 $\mathrm{m} / \mathrm{s})$, comes out very close to the mean velocity of blood flowing through the thoracic aorta of $\operatorname{dogs}(\approx 4.6 \mathrm{~m} / \mathrm{s})$ [1, [6]. Although this is a surprise agreement, considering the fact that our theory is valid only for a singlechannelled flow, while the experimental [6] measurement is for the total multi-channelled network, we feel that this happens because of the rather insulated nature of the thoracic aorta compared to its neighborhoods, which in effect, probably renders it as some approximation of a single-channelled tube. This seems an interesting comparison of our theory with experiments, although, at this stage, we would not dare any further conclusions based only on this information.

In Fig.I we show the variation of velocities (actually the scaled velocity difference, to facilitate plotting) of different modes with respect to the wave vector, where the corresponding parameters (mentioned above) are used.

From this figure, it appears that the velocity decreases with the increase of wave number, which is an obvious signature of the presence of viscous damping in the system. This information, although apparently a bit more on the mathematical side, in fact, can be of crucial importance to the medical person in analysing the exact location of the haemorrhagic spot. For this all that the doctor needs to know are the blood velocities at a few arbitrary spatial localions, in any major aorta or vein (say, 


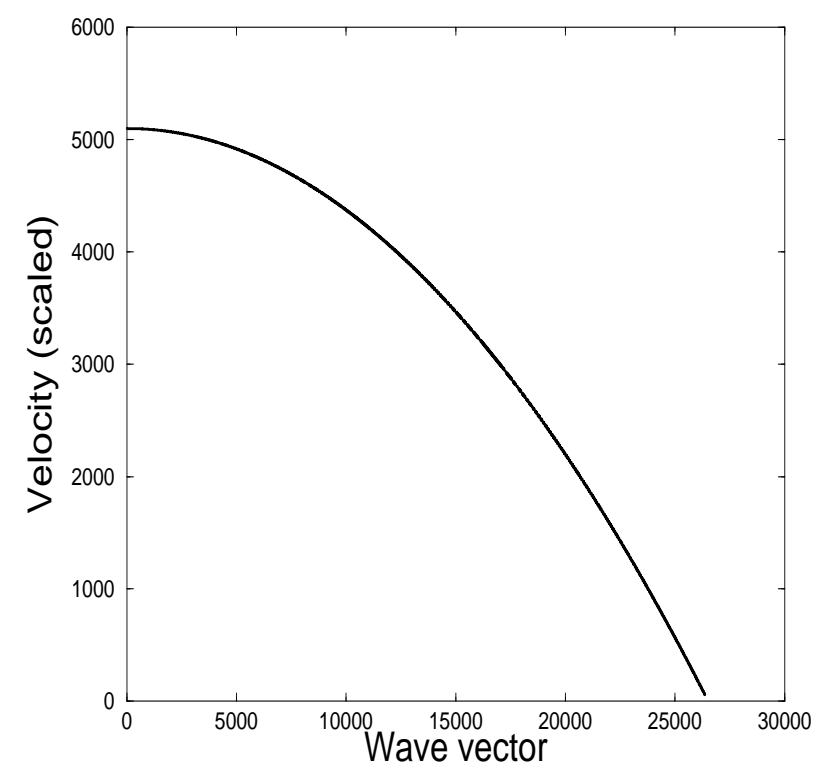

FIG. 1: Velocity vs wave vector plot, suitably scaled.

pulmonary). The remaining exercise would be a technical triviality where a graph is to be drawn plotting blood velocities against the corresponding spatial locations and these data would then be translated into the wave-vector space through a computer calibration for exact comparison with our theoretical results. Now whenever there is any aperiodicity in the blood flow, due to internal haemorrhagic clotting, the Fig.I. would show discontinuities in the form of spikes in the velocity spectrum, at the sites where haemorrhage has occurred. In the language of physics, these are nothing but effects of varying initial conditions originating from the affected sites. The fact that observation of the flow in any artery or vein is sufficient is directly related to the fact that the arterial or venal system is a multi-connected network, which means that the discontinuity arising in one branch will carry through to all subsequent branches. A following inspection of the graph will immediately give an idea on where this spot is to be expected, or at least a rough idea of the zone to operate on. Having said that, we should still be conscious of the fact that our theory presented here is basically for a pulsed flow in a single elastic tube. To have an exact comparison with the real biological system, we need to consider the multi-channelled branching of arteries (or veins), which certainly is a more complex network. The importance of our theory lies in the fact that to have an understanding of this multi-channelled network, we need to know the dynamics of each individual channel, after which an analysis of the apparently complicated network boils down to a simple enough initialvalue problem at each individual node, something which is easily solvable numerically.

In the following graphical illustrations, we consider two

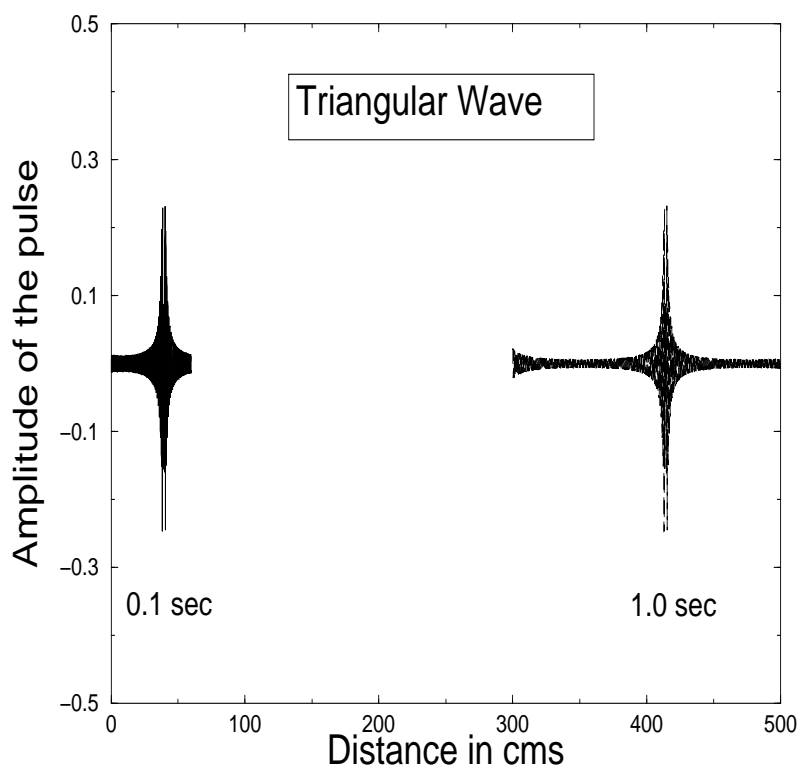

FIG. 2: Comparison between triangular wave pattern vs distance graphs after $0.1 \mathrm{sec}$ and $1.0 \mathrm{sec}$.

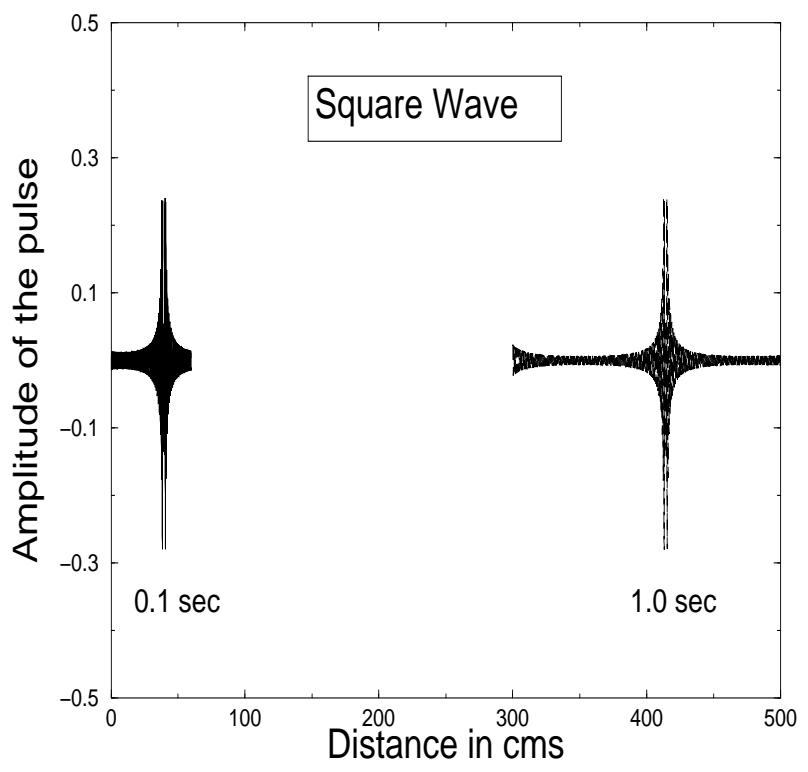

FIG. 3: Comparison between square wave pattern vs distance graphs after $0.1 \mathrm{sec}$ and $1 \mathrm{sec}$.

different initial conditions and follow their evolution with time as defined in eqn.(7), in a co-moving frame of reference.

Fig.II gives the comparison between the spatial variation of an input hypothetical triangular wave at times $0.1 \mathrm{sec}$ and $1 \mathrm{sec}$. These while obeying the same dynamics as wave pulses through an elastic tube, clearly show damping along the line of propagation.

Fig.III shows identical variations for an input square wave-train (hypothetical) at the same times previously 


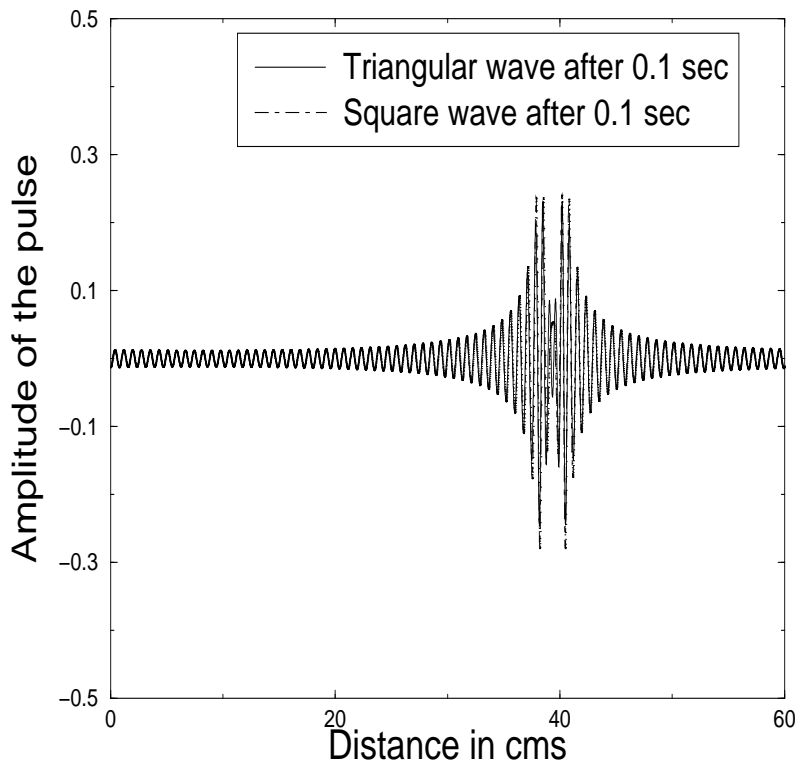

FIG. 4: Comparison between triangular and square wave pattern vs distance graphs after 0.1 sec. The two graphs are almost indistinguishable.

mentioned.

Finally, a comparison between two completely different hypothetical input pulses has been given in Fig.IV at 0.1 sec. which shows that although the pulses might start with widely varying initial conditions, the damping mechanism operating with non-zero viscosity leaves very little difference between their output waveforms after times of the order of $0.1 \mathrm{sec}$ (and larger times).

This study leads us to the conclusion that inspite of starting with widely varying initial conditions, the pressure pulses lose their memory very quickly and develop exactly identical forms. This happens because except a particular mode, the critical mode $k_{0}=\sqrt{\frac{\gamma}{\lambda}}$, in the Fourier spectrum, all other modes have non-vanishing attenuation. However, for small time evolution, in the scales of measurements ( $\sim$ millisecs $)$, the patterns are perfectly distinguishable and may be utilized in getting an idea of the variation of the mean viscosity of the blood to the local viscosity corresponding to a particular wave vector mode, from a knowledge of the critical mode.

\section{CONCLUSIONS AND DISCUSSIONS}

Our objective was to devise a theory which is capable, more or less exactly, to pinpoint the location of an internal haemorrhagic spot, in a single arterial or venal channel, due to clotting of blood. A knowledge of this can easily be obtained from Fig.I by comparing the variations of the velocity of the flow against the spatial locations at different points of any artery or vein, more technically speaking, by looking at the eventual discon- tinuities in the spectrum. The fact, that the attenuated waveforms become independent of the nature of the input waveforms, after times of the order of $0.1 \mathrm{sec}$, is proved by Figs.II-IV. This fact is of immense importance to our description, since, this in a way points to sort of an universality in the nature of our analysis. To be more specific, our analysis shows that we do not even need an exact description of the nature of the arterial or venal pulses in individuals to apply our theory. Rather the nature of the arterial (or venal) dynamics adjusts itself in such a self-consistent way that all specific input details are washed out and only the system elastic properties become the sole deciding parameter of the position of eventual blood clotting. The propagating pressure waves are defined only within a wave-vector window (eqn.(9)) and avoid possibilities of unwanted instabilities in eqn.(7). Incidentally, it might be noted that our theory is exact for blood plasma, which is a very close approximation to a Newtonian fluid, although, blood, in general has minor non-Newtonian characteristics. As results obtained from the model calculations show that our observation tallies extremely well with the experimental value of the velocity of blood flowing through the thoracic aorta of dogs, we have justified hopes that compared with real life situations, the location of the haemorrhagic spot, calculated from an extended version of our theory and including the multi-channelled branching of the arterial (or venal) network, would lie within acceptable error limits. However, as is the wont of any basic theory, ours too is wrought with a few characteristic assumptions as we have indicated earlier too. The theory presented here is certainly not complete enough for comparison with a real life experiment done on a multi-connected arterial (or venal) network, not at least at this stage. But our objective was basically to describe the dynamics of flow in each individual channel of the multi-connected network, which, with well defined initial conditions, could simulate the entire description of a real life flow. We look forward to experimental verifications of our theory, with experiments done on a single tube and designed essentially in the way we have described earlier in the text. For more realistic comparisons, we are working on a numerical model with all the multi-channelled subtleties involved [28], an essential coupling of single-channeled information analysed here to the generation of a multi-channelled network.

AKC acknowledges discussions with J. K. Bhattacharjee and C. Dasgupta during this work.

[1] Rubinow, S. I. (1975). Introduction to Mathematical Biology, John Wiley and Sons, and references therein.

[2] McDonald, D. A. (1965). Blood Flow in Arteries, 2nd ed., Williams and Wilkin, Baltimore.

[3] Rubinow, S. I. and Keller, J. B. (1972). Flow of a Viscous Fluid Through an Elastic Tube with Applications 
to Blood Flow. J. Theo. Biol. 35, 299-313.

[4] Brecher, G. A. (1952). Mechanism of venous flow under different degrees of aspiration. Amer. J. Physiology 169, 423-433.

[5] Bergel, D. H. (1961). The static elastic properties of the arterial wall. J. Physiology 156, 445-457.

[6] McDonald, D. A. (1968). Regional pulse-wave velocity in the arterial tree. J. Appl. Physiology 24, 73-78.

[7] Fung, Y. C. (1990). Biomechanics: Motion, Flow, Stress, and Growth, Springer-Verlag, NY, and references therein.

[8] Alstrom, P. et al (1995). Instability and "Sausage-String" Appearance in Blood Vessels during High Blood Pressure. Phys. Rev. Lett. 82, 1995-1998.

[9] Gustaffson, F. (1997). Hypertensive Arteriolar Necrosis Revisited. Blood Pressure 6, 71-77.

[10] Bar-Ziv, R. and Moses, E. (1994). Instability and "Pearling" States Produced in Tubular Membranes by Competition of Curvature and Tension. Phys. Rev. Lett. 73, 1392-1395.

[11] Wessel, N. et al (2000). Short-term forecasting of lifethreatening cardiac arrhythmias based on symbolic dynamics and finite-time growth rates. Phys. Rev. E 61, 733-739 and references therein.

[12] Liebovitch, L. S. et al (1999). Nonlinear properties of cardiac rhythm abnormalities. Phys. Rev. E 59, 33123319 and references therein.

[13] Fenton, F. H., Evans, S. J. and Hastings H. M. (1999). Memory in an Excitable Medium: A Mechanism for Spiral Wave Breakup in the Low-Excitability Limit. Phys. Rev. Lett. 83, 3964-3967.

[14] Fenton, F. and Karma, A. (1998). Fiber-RotationInduced Vortex Turbulence in Thick Myocardium. Phys. Rev. Lett. 81, 481-484.

[15] Sinha, S., Pande, A. and Pandit, R. (2001). Defibrillation via the Elimination of Spiral Turbulence in a Model for Ventricular Fibrillation. Phys. Rev. Lett. 86, 36783681; Das, B. and Batra, R. L. (1995). Non-Newtonian flow of blood in an arteriosclerotic blood vessel with rigid permeable walls. J. Theo. Biol. 175, 1-11.

[16] Hastings, H. M. et al (2000). Alternans and the onset of ventricular fibrillation. Phys. Rev. E 62, 4043-4048.
[17] Narayanan, K. et al (1998). Unstable periodic orbits in human cardiac rhythms. Phys. Rev. E 57, 4594-4603.

[18] Panfilov, A. V. et al (2000). Elimination of spiral waves in cardiac tissue by multiple electrical shocks. Phys. Rev. E 61, 4644-4647.

[19] Rappel, W. J., Fenton, F. and Karma, A. (1999). Spatiotemporal Control of Wave Instabilities in Cardiac Tissue. Phys. Rev. Lett. 83, 456-459.

[20] Xie, F., Qu, Z. and Garfinkel, A. (1998). Dynamics of reentry around a circular obstacle in cardiac tissue. Phys. Rev. E 58, 6355-6358.

[21] Amaral, L. A. N. et al (1998). Scale-Independent Measures and Pathologic Cardiac Dynamics. Phys. Rev. Lett. 81, 2388-2391; Amaral, L. A. N. et al (2001). BehavioralIndependent Features of Complex Heartbeat Dynamics. Phys. Rev. Lett. 86, 6026-6029.

[22] Lin, D. C. and Hughson, R. L. (2001). Modeling Heart Rate Variability in Healthy Humans: A Turbulence Analogy. Phys Rev. Lett. 86, 1650-1653.

[23] Frohlinde, V. S. et al (2001). Noise Effects on the Complex Patterns of Abnormal Heartbeats. Phys. Rev. Lett. 87, 068104.

[24] Folkow, B. and Neil, E. (1971). Circulation, Oxford University Press, New York.

[25] Landau, L. D. and Lifshitz, E. M. (1981). Fluid Mechanics Butterworth-Heinemann Ltd.

[26] Reynold's number, $R e=\frac{v L}{\nu / \rho}$, where $v$ is the velocity of the flow and $L$ is the length scale of the geometry of the flow, $\nu / \rho$ being the kinematic viscosity. For blood flowing through the aorta of a human body, $v \sim 30 \mathrm{~cm} / \mathrm{secs}$. 24] and from Fig.IV we see that even at the order of 0.1 sec, the solutions remain initial condition independent. So we chose $\mathrm{t}=0.001 \mathrm{sec}$ and putting in the values of the parameters, it can be easily shown that $R e<60$.

[27] Henry, H. and Hakim, V. (2000). Linear Stability of Scroll Waves. Phys. Rev. Lett. 85, 5328-5331; Barkley, D. (1994). Euclidean symmetry and the dynamics of rotating spiral waves. Phys. Rev. Lett. 72, 164-167.

[28] Chattopadhyay, A. K. (unpublished). 